\title{
Physical capacity, occupational physical demands, and relative physical strain of older employees in construction and healthcare
}

\author{
Suzanne L. Merkus ${ }^{1} \mathbb{D} \cdot$ Lars-Kristian Lunde $^{1} \cdot$ Markus Koch $^{1} \cdot$ Morten Wærsted $^{1}$ (D) Stein Knardahl $^{1}$.D \\ Kaj Bo Veiersted ${ }^{1}$ (D)
}

Received: 2 May 2018 / Accepted: 29 October 2018 / Published online: 15 November 2018

(c) The Author(s) 2018

\begin{abstract}
Purpose To assess age-related differences in physical capacity, occupational physical demands, and relative physical strain at a group level, and the balance between capacity and demands at an individual level, for construction and healthcare workers. Methods Shoulder strength, back strength, and aerobic capacity were assessed among construction $(n=62)$ and healthcare workers $(n=64)$. During a full working day, accelerometers estimated upper-arm elevation, trunk flexion, and occupational physical activity as indicators of occupational physical demands. Simultaneously, normalised surface electromyography $\left(\% \mathrm{sEMG}_{\max }\right)$ of the upper trapezius and erector spinae muscles, and normalised electrocardiography (percentage heart rate reserve (\%HRR)) estimated relative physical strain. Differences between younger ( $\leq 44$ years) and older ( $\geq 45$ years) workers, as well as the moderating effect of age on the associations between capacity and demands, were analysed per sector.

Results Compared to younger workers, older workers had similar strength and lower aerobic capacity; older construction workers had similar demands while older healthcare workers had higher demands. Compared to younger workers, older employees had unfavourable muscle activity patterns; \%HRR had a tendency to be lower for older construction workers and higher for older healthcare workers. Among construction workers, age moderated the associations between shoulder strength and arm elevation $(p=0.021)$, and between aerobic capacity and occupational physical activity $(p=0.040)$. Age did not moderate these associations among healthcare workers.

Conclusions In both sectors, the level of occupational physical demands and the higher relative physical strain in older employees require addressing to promote sustainable work participation among an aging population.
\end{abstract}

Keywords Aging $\cdot$ Muscle strength $\cdot$ Aerobic capacity $\cdot$ Electromyography $\cdot$ Electrocardiography $\cdot$ Inclinometry

\section{Introduction}

Employees in physically demanding occupations, such as in construction and healthcare, are at increased risk for longterm sick leave, disability pensioning, and early retirement (Labriola et al. 2009; Sundstrup et al. 2018). The physical demands of construction and healthcare work, together with the high prevalence rates of musculoskeletal disorders in

Electronic supplementary material The online version of this article (https://doi.org/10.1007/s00420-018-1377-5) contains supplementary material, which is available to authorized users.

Suzanne L. Merkus

suzanne.merkus@stami.no

1 National Institute of Occupational Health, $\mathrm{Pb} 5330$

Majorstuen, 0304 Oslo, Norway these sectors (Davis and Kotowski 2015; Umer et al. 2017), are factors that influence the ability to sustain work until retirement age (Jarvholm et al. 2014; Jebens et al. 2014; Jensen et al. 2012; Oude Hengel et al. 2012). As retirement age increases in many countries, insight into determinants of sustainable employability gain importance.

Sustaining employment in physically demanding occupations is largely determined by the balance between physical capacity and occupational physical demands (de Zwart et al. 1995). As a natural process of aging, physical capacity declines with age (Hamberg-van Reenen et al. 2009). This leads to an age-related imbalance between capacity and demands if the level of occupational physical demands is maintained with age. In this situation, older employees will work closer to their maximum capacity and relative physical strain will be higher (de Zwart et al. 1995; Holtermann et al. 2018). Therefore, several studies suggest that lowering 
physical demands for older employees is crucial to sustaining employment (Jarvholm et al. 2014; Jebens et al. 2014).

Conceptually, age-related changes in the balance between capacity and demands, and the effects on relative strain are straightforward. However, some inconsistencies in the literature regarding comparisons between older and younger employees in physically demanding occupations suggest that the relationships are not so clear-cut. First, although physical capacity declines as a natural process of aging (Hamberg-van Reenen et al. 2009; Soer et al. 2012), some studies among older employees in physically demanding occupations have found that capacity is maintained with age (Gall and Parkhouse 2004; Schibye et al. 2001; Torgen et al. 1999). Second, the recommendation of lowering occupational physical demands with age is not always followed (Aittomaki et al. 2005; Burr et al. 2017; Jarvholm et al. 2014; Jebens et al. 2014; Tonnon et al. 2017). Third, both decreases and increases in relative physical strain with age have been reported (Brighenti-Zogg et al. 2016; Gupta et al. 2014; Jebens et al. 2015).

These inconsistencies may, in part, be attributed to the use of different assessment methods, including subjective assessments and observation (Aittomaki et al. 2005; Burr et al. 2017; Rasmussen et al. 2015; Torgen et al. 1999). These methods are characterised by recall and (self-)estimation bias; for valid assessments, objective measures are preferred (Koch et al. 2016). Additionally, comparisons between older and younger workers at the group level does not provide insight into age-related differences in the balance between physical capacity and occupational physical demands at an individual level. Insight into valid levels of physical capacity, occupational physical demands, and relative physical strain among older employees at a group level, as well as insight into the balance between capacity and demands at an individual level, will improve recommendations and interventions that promote sustainable work participation.

Therefore, using objective assessments, we asked the following research questions for construction and healthcare workers: (1) are there differences between older and younger employees in physical capacity, occupational physical demands, and relative physical strain? (2) Does the association (i.e., the balance) between physical capacity and occupational physical demands depend on age?

\section{Methods}

\section{Participants and procedures}

This cross-sectional study is part of a previously described larger prospective cohort study (Koch et al. 2016; Lunde et al. 2014). From a sample of 594 construction and healthcare workers (response rate 51\%) who filled in a questionnaire at baseline, 371 workers consented to participate in objective assessments. From this sample, 66 construction and 72 healthcare workers were selected based on logistics (availability, age, and occupational titles). Exclusion criteria were a diagnosis of cardiovascular disease or pregnancy. All participants underwent a physical examination by a physician or nurse a few days prior to the start of the objective assessments. Simultaneous recordings of occupational physical demands and relative physical strain were done during a full $8 \mathrm{~h}$ working day.

\section{Assessment methods}

A comprehensive summary of the methods is provided below; a more in-depth description of the methods is provided in the Supplementary file.

\section{Physical capacity}

Indicators of physical capacity were physical strength and aerobic capacity. Physical strength was assessed as isometric shoulder strength $(\mathrm{kg})$, tested in a standardized seated position, and isometric back extension strength $(\mathrm{kg})$, tested using a modified Biering-Sørensen test (Bieringsorensen 1984). For each test, the highest value of three attempts was used as maximal voluntary contraction (MVC). A submaximal test on a cycle ergometer estimated aerobic capacity $\left(V \mathrm{O}_{2 \max }\right.$ in $\mathrm{L} / \mathrm{kg} / \mathrm{min}$ ) (Astrand 2003). For further information see the Supplementary file and Lunde et al. (2014).

\section{Occupational physical demands}

Indicators of occupational physical demands were upper-arm elevation, trunk flexion, and occupational physical activity (OPA). Accelerometers were used to estimate these indicators. The duration (percentage of the working day) and frequency (average number per hour) of upper-arm elevations and trunk flexions $>30^{\circ}$ and $>60^{\circ}$ were calculated (Coenen et al. 2016). OPA was calculated as the duration (percentage of the working day) spent standing, moving, and walking (Skotte et al. 2014). See also the Supplementary file and Lunde et al. (2014).

\section{Relative physical strain}

Indicators of relative physical strain were the percentage of the normalised surface electromyography $\left(\% \mathrm{sEMG}_{\max }\right)$ and the percentage of the heart rate reserve (\%HRR). Surface electromyography (sEMG) recorded muscle activity of the dominant shoulder (upper trapezius muscle) and of the lower back (both erector spinae longissimus muscles). Recordings were processed and normalised to the maximal muscle activity $\left(\mathrm{sEMG}_{\max }\right)$ assessed during the 
strength tests (Mathiassen et al. 1995). The percentages of the working day with muscle activity $>15 \% \mathrm{sEMG}_{\max }$ (high strain) (Anton et al. 2003; Mathiassen and Winkel 1991) and $<0.5 \% \mathrm{sEMG}_{\max }$ (muscular rest) (Veiersted et al. 1993) were calculated. Additional indicators were the median load level (50th percentile of the amplitude probability distribution function (APDF)); the peak load level (90th percentile APDF); and the frequency (average number per hour) with very high strain activity $>63 \%$ $\mathrm{sEMG}_{\max }$ (Mathiassen and Winkel 1991).

Electrocardiography monitored heart rate and \%HRR was calculated using the following equation $\left(\% \mathrm{HRR}=\frac{\mathrm{HR}-\mathrm{HR}_{\min }}{\mathrm{HR}_{\max }-\mathrm{HR}_{\min }} \times 100 \%\right)$. Maximal heart rate $\left(\mathrm{HR}_{\max }\right)$ was estimated by $208-(0.7 \times$ age) (Tanaka et al. 2001). Minimum heart rate $\left(\mathrm{HR}_{\mathrm{min}}\right)$ was defined as the minimum value of a running average of 10 beats during the waking period of all 3-4 assessment days. Average \%HRR and the percentage of the working day $>33 \%$ HRR (high strain) were calculated (Gupta et al. 2014). For more information see the Supplementary file and Lunde et al. (2014).

\section{Demographic, health-related, and work-related information}

Participants filled in a questionnaire on demographic, healthrelated, and work-related information. Based on previous research among aging employees, age was categorised into $\leq 44$ and $\geq 45$ years (Burr et al. 2017; Schibye et al. 2001; Soer et al. 2012). The questionnaire further included gender, body mass index (BMI), and general health status (SF36 single-item) (Ware 2000). Pain intensity was assessed for the dominant shoulder and lower back (Kuorinka et al. 1987). Leisure time physical activity and the pursuit of exercises to prevent or treat complaints of the musculoskeletal system were each assessed with a single question. Workrelated information included weekly working hours, subjective work ability (Work Ability Index single-item) (Ahlstrom et al. 2010), and subjectively experienced physical work heaviness. Occupational title was categorised according to the level of physical demands into sedentary/light work, medium intensity work, and heavy work as suggested by the Dictionary of Occupational Titles (DOT) (U.S. Department of Labor 1991) and confirmed via visual inspection of our objective assessments. Subscales of the General Questionnaire for Psychological and Social Factors at Work $\left(\mathrm{QPS}_{\text {Nordic }}\right)$ assessed job demands (including questions on work pace, overtime, and the amount of work) and control at work (Dallner et al. 2000). A single item from the QPS $_{\text {Nordic }}$ assessed whether employees noticed inequalities in how older and younger employees are treated at the workplace. For more detail, see the Supplementary file.

\section{Data analysis}

Analyses were conducted in IBM SPSS (25.0). Statistical significance was set at $p<0.05$. Due to differences in occupational physical demands between the construction and healthcare sectors, all analyses were stratified for sector. Differences between the age groups (i.e., $\leq 44$ years and $\geq 45$ years) for demographic, health-related, and subjective work-related variables, as well as for physical capacity, occupational physical demands, and relative physical strain indicators were tested with Fisher's exact tests for categorical variables, $t$ tests for normally distributed continuous variables, and Mann-Whitney $U$ tests for skewed continuous variables. Sensitivity analyses assessed age-related differences in physical capacity, occupational physical demands, and relative physical strain for employees at increased risk of early exit from work, i.e., for employees with occupations that are medium and heavy according to DOT.

Regression analyses studied whether the association between occupational physical demands and physical capacity depended on age, i.e., whether age had a moderating effect on the association. Non-normally distributed dependent variables were transformed. Dependent variables were duration of arm elevation $>60^{\circ}$ (natural logarithm transformation), duration of trunk flexion $>60^{\circ}$, and duration standing/moving/walking (squared transformation). Regression coefficients for arm elevation were back transformed $\left(e^{b}=\right.$ $\operatorname{Exp}(b))$, as were coefficients for standing/moving/walking (square $\operatorname{root}(b)=\operatorname{Sqrt}(b))$. For each dependent variable, the respective capacity indicator, i.e., shoulder strength, back strength, and aerobic capacity, were added as independent variables, together with age ( $\leq 44$ and $\geq 45)$, and the interaction between age and the capacity indicator. Additional analyses identified explanatory variables of interaction effects. Potential variables were chosen based on logic and prior research: gender, general health, pain, preventative exercises, leisure time physical activity, psychosocial work factors, work ability, and level of physical demands (DOT). A variable was considered to be explanatory when an interaction changed by $>10 \%$ after adding the variable to the model.

\section{Results}

From the 138 selected participants, 12 employees were unable to participate due to various practical reasons, leading to a final study sample of 126 employees (construction $n=62$; healthcare $n=64$ ). The construction participants included project managers, supervisors, engineers, bricklayers, carpenters, concrete workers, and assistants. The healthcare participants included managers, nurses, nursing assistants, social educators, and kitchen and cleaning staff. Data from accelerometers were available from 92 to $95 \%$ of the 
participants (arm elevation $n=120$; trunk flexion $n=115$; activities $n=119)$. sEMG data for the upper trapezius muscle were available from $75 \%$ of the participants (construction $n=39$; healthcare $n=56$ ), and for the erector spinae muscles from $40 \%$ of the participants (construction $n=22$; healthcare $n=28$ ). Heart rate data were available from $81 \%$ of the participants (construction $n=46$; healthcare $n=56$ ). From the questionnaire, nine out of the 14 questions used in this study missed $2-19 \%$ of the answers; the remaining questions were complete.

The average age for construction workers was 39.9 years (SD 13.4 years; range 19-67 years), and for healthcare workers 44.1 years (SD 9.9 years; range 20-64 years). The sample of construction workers consisted of 61 males and one female, while one-fifth of the healthcare workers were male (Table 1). Both the construction and healthcare samples included occupations with sedentary/light physical demands according to the DOT classification; however, medium intensity work was not represented among construction workers, and heavy intensity work was not represented among healthcare workers. There was a tendency that older construction workers more often had sedentary/ light physical work. Compared to their younger colleagues, older healthcare workers were more likely to perceive their work as moderate to somewhat heavy $(p=0.013)$. In both sectors, younger workers reported that older and younger workers were not treated equally; the difference between the age groups reached statistical significance for construction workers $(p<0.001)$ (Table 1$)$.

\section{Physical capacity}

Due to the strong dependency on sex, physical strength and aerobic capacity are reported separately for male and female workers (Table 2). Construction workers $\geq 45$ years had lower aerobic capacity than male construction workers $\leq 44$ years $(p=0.001)$; shoulder and back strength were similar between the age groups. Compared to their younger colleagues, male and female healthcare workers $\geq 45$ years had lower aerobic capacity; however, the differences did not reach statistical significance.

\section{Occupational physical demands}

For construction workers, no statistically significant age differences were found for duration or frequency of arm elevation and trunk flexion $>30^{\circ}$ and $>60^{\circ}$, nor for duration of OPA, although older workers had a tendency for shorter duration of trunk flexion $>60^{\circ}$ than younger workers (Table 3).

With exception of duration of arm elevation $>30^{\circ}$, older healthcare workers ( $\geq 45$ years) had statistically significantly higher duration and frequency of arm elevation and trunk flexion $>30^{\circ}$ and $>60^{\circ}$ than younger healthcare workers ( $\leq 44$ years) (Table 3 ). There was no statistically significant difference between the age groups for OPA (Table 3 ).

\section{Relative physical strain}

Older construction workers had statistically significantly longer duration of upper trapezius muscle activity $>15 \%$ $\mathrm{sEMG}_{\mathrm{max}}$, shorter duration of muscle rest, higher median and peak load levels, and a higher frequency of activity $>63 \%$ $\mathrm{sEMG}_{\max }$ than their younger colleagues (Table 4). A similar increased tendency of erector spinae muscle activity was seen amongst construction workers $\geq 45$ years; however, differences between the age groups were not statistically significant (Table 4). Compared to younger construction workers, workers $\geq 45$ years had a lower average $\%$ HRR and spent less time $>33 \%$ HRR; however, differences did not reach statistical significance (Table 4).

Older healthcare workers had statistically significantly longer duration of upper trapezius muscle activity $>15 \%$ $\mathrm{sEMG}_{\max }$, as well as a higher peak load level and frequency of activity $>63 \% \mathrm{sEMG}_{\max }$ than their younger colleagues (Table 4). All parameters of the erector spinae muscles were statistically significantly higher for older compared to younger healthcare workers (Table 4). Increases in average $\%$ HRR and duration $>33 \%$ HRR for healthcare workers $\geq 45$ years compared to those $\leq 44$ years were not statistically significant (Table 4).

\section{Sensitivity analyses}

The sensitivity analyses that included medium and heavy occupations only, resulted in two deviations from the analyses involving the whole sample (See Tables 1, 2, 3 in the Supplementary file). First, average \%HRR $(p=0.021)$ and duration $>33 \%$ HRR $(p=0.009)$ were statistically significantly lower for older than younger constructions workers. Second, arm elevation $>60^{\circ}$ regarding duration $(p=0.066)$ and frequency $(p=0.081)$ were no longer statistically significantly different for older and younger healthcare workers.

\section{Association between capacity and demands in relation to age}

Among construction workers, age modified the associations between shoulder strength and duration of arm elevation $>60^{\circ}$ (shoulder strength $\times$ age $b=1.07 ; p=0.021$ ), and between aerobic capacity and OPA (aerobic capacity $\times$ age $b=15.21 ; p=0.040$ ) (Table 5). The positive interaction effects indicated that construction employees $\geq 45$ years were more likely to have a better balance between arm elevation $>60^{\circ}$ and shoulder strength, and between OPA and aerobic capacity, than employees $\leq 44$ years (Fig. 1). In the 
Table 1 Demographic, health-related, and subjective work-related information for older ( $\geq 45$ years) and younger ( $\leq 44$ years) employees in construction and healthcare

\begin{tabular}{|c|c|c|c|c|c|c|c|c|}
\hline & \multicolumn{4}{|c|}{ Construction } & \multicolumn{4}{|l|}{ Healthcare } \\
\hline & $\begin{array}{l}\text { Total } \\
n=62\end{array}$ & $\begin{array}{l}\leq 44 \text { years } \\
n=39\end{array}$ & $\begin{array}{l}\geq 45 \text { years } \\
n=23\end{array}$ & $p$ & $\begin{array}{l}\text { Total } \\
n=64\end{array}$ & $\begin{array}{l}\leq 44 \text { years } \\
n=33\end{array}$ & $\begin{array}{l}\geq 45 \text { years } \\
n=31\end{array}$ & $p$ \\
\hline & $n(\%)$ & $n(\%)$ & $n(\%)$ & & $n(\%)$ & $n(\%)$ & $n(\%)$ & \\
\hline \multicolumn{9}{|l|}{ Gender } \\
\hline Male & $61(98)$ & $38(97)$ & $23(100)$ & 0.629 & $14(22)$ & $8(24)$ & $6(19)$ & 0.337 \\
\hline Female & $1(2)$ & $1(3)$ & $0(0)$ & & $50(78)$ & $25(76)$ & $25(81)$ & \\
\hline \multicolumn{9}{|l|}{ Level of physical demands (DOT) } \\
\hline Sedentary/light work & $16(26)$ & $8(21)$ & $8(35)$ & 0.242 & $8(13)$ & $5(15)$ & $3(10)$ & 0.709 \\
\hline Medium intensity work & - & - & - & & $56(87)$ & $28(85)$ & $28(90)$ & \\
\hline Heavy work & $46(74)$ & $31(79)$ & $15(65)$ & & - & - & - & \\
\hline \multicolumn{9}{|l|}{ General health } \\
\hline Very good to excellent & $28(45)$ & $21(54)$ & $7(30)$ & 0.192 & $30(47)$ & $14(42)$ & $16(52)$ & 0.568 \\
\hline Good & $26(42)$ & $14(36)$ & $12(52)$ & & $25(39)$ & $13(40)$ & $12(38)$ & \\
\hline Poor to fair & $8(13)$ & $4(10)$ & $4(18)$ & & $9(14)$ & $6(18)$ & $3(10)$ & \\
\hline \multicolumn{9}{|l|}{ BMI } \\
\hline Normal $\left(<25 \mathrm{~kg} / \mathrm{m}^{2}\right)$ & $25(40)$ & $17(44)$ & $8(35)$ & 0.596 & $34(53)$ & $20(61)$ & $14(45)$ & 0.316 \\
\hline Overweight $\left(>25 \mathrm{~kg} / \mathrm{m}^{2}\right)$ & $37(60)$ & $22(56)$ & $15(65)$ & & $30(47)$ & $13(39)$ & $17(55)$ & \\
\hline \multicolumn{9}{|l|}{ Shoulder pain } \\
\hline No pain—a little pain & $50(85)$ & $32(87)$ & $18(82)$ & 0.715 & $40(67)$ & $16(53)$ & $24(80)$ & 0.054 \\
\hline Moderate—severe pain & $9(15)$ & $5(13)$ & $4(18)$ & & $20(33)$ & $14(47)$ & $6(20)$ & \\
\hline \multicolumn{9}{|l|}{ Lower back pain } \\
\hline No pain—a little pain & $45(74)$ & $30(79)$ & $15(65)$ & 0.368 & $40(65)$ & $18(56)$ & $22(73)$ & 0.192 \\
\hline Moderate—severe pain & $16(26)$ & $8(21)$ & $8(35)$ & & $22(35)$ & $14(44)$ & $8(27)$ & \\
\hline \multicolumn{9}{|l|}{ Subjective work demands } \\
\hline Not at all—light & $15(24)$ & $8(21)$ & $7(30)$ & 0.177 & $21(33)$ & $14(44)$ & $7(23)$ & 0.013 \\
\hline Moderate_-somewhat heavy & $31(50)$ & $23(58)$ & $8(35)$ & & $27(43)$ & $8(25)$ & $19(61)$ & \\
\hline Heavy_very, very heavy+ & $15(26)$ & $8(21)$ & $8(35)$ & & $15(24)$ & $10(31)$ & $5(16)$ & \\
\hline \multicolumn{9}{|l|}{ Preventative exercises } \\
\hline No & $21(34)$ & $12(31)$ & $9(39)$ & 0.583 & $13(20)$ & $7(21)$ & $6(19)$ & 1.000 \\
\hline Yes & $41(66)$ & $27(69)$ & $14(61)$ & & $51(80)$ & $26(79)$ & $25(81)$ & \\
\hline \multicolumn{9}{|l|}{ Leisure physical activity } \\
\hline Sedentary—some activity & $40(71)$ & $22(67)$ & $18(78)$ & 0.385 & $38(73)$ & $16(64)$ & $22(82)$ & 0.215 \\
\hline \multirow[t]{4}{*}{ Regular-hard activity } & $16(29)$ & $11(33)$ & $5(22)$ & & $14(27)$ & $9(36)$ & $5(18)$ & \\
\hline & \multicolumn{4}{|c|}{ Construction } & \multicolumn{4}{|l|}{ Healthcare } \\
\hline & $\begin{array}{l}\text { Total } \\
n=62\end{array}$ & $\begin{array}{l}\leq 44 \text { years } \\
n=39\end{array}$ & $\begin{array}{l}\geq 45 \text { years } \\
n=23\end{array}$ & $p$ & $\begin{array}{l}\text { Total } \\
n=64\end{array}$ & $\begin{array}{l}\leq 44 \text { years } \\
n=33\end{array}$ & $\begin{array}{l}\geq 45 \text { years } \\
n=31\end{array}$ & $p$ \\
\hline & $m(\mathrm{SD})$ & $m(\mathrm{SD})$ & $m(\mathrm{SD})$ & & $m(\mathrm{SD})$ & $m(\mathrm{SD})$ & $m(\mathrm{SD})$ & \\
\hline Age (years) & $39.9(13.4)$ & $31.0(6.5)$ & $55.1(6.7)$ & $<0.001$ & $44.1(9.9)$ & $36.3(6.0)$ & $52.5(5.1)$ & $<0.001$ \\
\hline Weekly working hours & $37.8(4.0)$ & $37.6(5.0)$ & $38.2(1.1)$ & 0.542 & $35.7(4.2)$ & $35.7(3.3)$ & $35.6(5.0)$ & 0.919 \\
\hline Work ability $(0-10)$ & $8.9(1.5)$ & $8.5(1.7)$ & $9.4(0.8)$ & 0.054 & $8.8(1.3)$ & $8.7(1.2)$ & $8.9(1.5)$ & 0.398 \\
\hline QPS job demands (1-5) & $3.0(0.6)$ & $3.1(0.5)$ & $2.9(0.6)$ & 0.276 & $3.0(0.7)$ & $3.0(0.8)$ & $3.0(0.7)$ & 0.814 \\
\hline QPS job control (1-5) & $3.2(0.7)$ & $3.2(0.7)$ & $3.3(0.8)$ & 0.483 & $2.9(0.8)$ & $3.0(0.8)$ & $2.8(0.7)$ & 0.454 \\
\hline Age inequality (1-5) & $2.1(0.9)$ & $2.4(0.8)$ & $1.6(0.7)$ & $<0.001$ & $2.1(1.2)$ & $2.4(1.4)$ & $1.8(0.9)$ & 0.088 \\
\hline
\end{tabular}

Bold typeface indicates statistically significant differences between age groups at $p<0.05$ 
Table 2 Physical capacity estimated by shoulder strength, back strength, and aerobic capacity for older $(\geq 45$ years $)$ and younger ( $\leq 44$ years) construction and healthcare workers, stratified by gender

\begin{tabular}{|c|c|c|c|c|c|c|c|c|c|}
\hline & \multicolumn{3}{|c|}{ Shoulder strength (kg) } & \multicolumn{3}{|c|}{ Back strength (kg) } & \multicolumn{3}{|c|}{$V \mathrm{O}_{2 \max }(\mathrm{L} / \mathrm{min} / \mathrm{kg})$} \\
\hline & $n$ & $m(\mathrm{SD})$ & $p$ & $n$ & $m(\mathrm{SD})$ & $p$ & $n$ & $m(\mathrm{SD})$ & $p$ \\
\hline \multicolumn{10}{|l|}{ Construction } \\
\hline \multicolumn{10}{|l|}{ Male $(n=61)$} \\
\hline$\leq 44$ years & 38 & $28.2(6.9)$ & & 38 & $48.4(14.8)$ & & 34 & $41.3(12.2)$ & \\
\hline$\geq 45$ years & 23 & $28.2(9.7)$ & 0.990 & 21 & $49.0(17.5)$ & 0.879 & 22 & $33.1(5.2)$ & 0.001 \\
\hline \multicolumn{10}{|l|}{ Healthcare } \\
\hline \multicolumn{10}{|l|}{ Male $(n=14)$} \\
\hline$\leq 44$ years & 8 & $24.2(5.2)$ & & 8 & $47.1(23.0)$ & & 7 & $38.7(13.1)$ & \\
\hline$\geq 45$ years & 6 & $24.4(6.9)$ & 0.953 & 6 & $38.6(13.8)$ & 0.440 & 6 & $26.7(7.2)$ & 0.071 \\
\hline \multicolumn{10}{|c|}{ Female $(n=50)$} \\
\hline$\leq 44$ years & 25 & $15.4(3.6)$ & & 24 & $30.2(10.2)$ & & 24 & $33.4(9.0)$ & \\
\hline$\geq 45$ years & 25 & $13.6(4.4)$ & 0.108 & 25 & $26.9(10.7)$ & 0.275 & 23 & $29.0(5.1)$ & 0.091 \\
\hline
\end{tabular}

Bold typeface indicates statistically significant differences between age groups at $p<0.05$ analyses that identified explanatory variables of the interaction effects, the largest change-and thereby best explanatory effect-was obtained by adding the categorical level of physical work demands (DOT) to the models. After adding the categorical level of physical work demands (DOT) to the models, the interactions were no longer statistically significant (shoulder strength $\times$ age $p=0.218$; aerobic capacity $\times$ age $p=0.734$ ) (Table 5). The association between back strength and the duration of trunk flexion $>60^{\circ}$ was not modified by age in construction workers (back strength $\times$ age $p=0.338$ ) (Table 5). For healthcare workers, age did not modify the associations between shoulder strength and duration of arm elevation $>60^{\circ}$ (shoulder strength $\times$ age $p=0.214$ ); between back strength and duration of trunk flexion $>60^{\circ}$ (back strength $\times$ age $p=0.760$ ); nor between aerobic capacity and OPA (aerobic capacity $\times$ age $p=0.686$ ) (Table 5; Fig. 1).

\section{Discussion}

This study assessed age-related differences in physical capacity, occupational physical demands, and relative physical strain at a group level, as well as age-related differences in the balance between capacity and demands at an individual level among construction and healthcare workers. Compared to their younger colleagues, older construction and healthcare workers had similar physical strength yet poorer aerobic capacity. Older workers had similar or higher occupational physical demands compared to younger workers. Relative physical strain, estimated by the pattern of relative muscle activity in the shoulder and lower back, was unfavourable for older workers. Relative physical strain, estimated by percentage of the heart rate reserve, had a tendency to be lower for older construction workers, specifically for those with heavy physical demands, and had a tendency to be higher for older healthcare workers. At an individual level, older employees in construction were more likely to have a better balance between capacity and demands compared to their younger colleagues. This was explained by the higher percentage of older participants having sedentary/ light work. For healthcare workers, the balance between capacity and demands at an individual level was similar for both age groups.

Studies of the general population (Soer et al. 2012) and of various physically demanding occupations (Nygard et al. 1991) suggest that both strength and aerobic capacity decline with age. Contrastingly, our study showed that only aerobic capacity was lower among older workers, while strength was similar for older and younger workers. These findings in our sample of construction and healthcare workers are consistent with findings among male power line technicians (Gall and Parkhouse 2004) and waste collectors (Schibye et al. 2001). This may suggest that some physically demanding work may contribute to maintaining strength, but not aerobic capacity (Gall and Parkhouse 2004; Jebens et al. 2015; Schibye et al. 2001; Soer et al. 2012; Torgen et al. 1999).

The similar or higher levels of occupational physical demands among the older construction and healthcare workers in our sample is not in line with recommendations to reduce demands as employees age (Aittomaki et al. 2005; Burr et al. 2017; Jarvholm et al. 2014; Jebens et al. 2014). This is of concern, because the level of occupational physical demands measured in our sample have previously been associated with musculoskeletal disorders (Coenen et al. 2016) and occupational physical demands may have stronger negative health effects for older employees than for younger employees (Burr et al. 2017). Therefore, the level of occupational physical demands among older construction and healthcare workers requires attention to preserve health and promote sustainable work participation until retirement age (Jebens et al. 2014; Oude Hengel et al. 2012). 


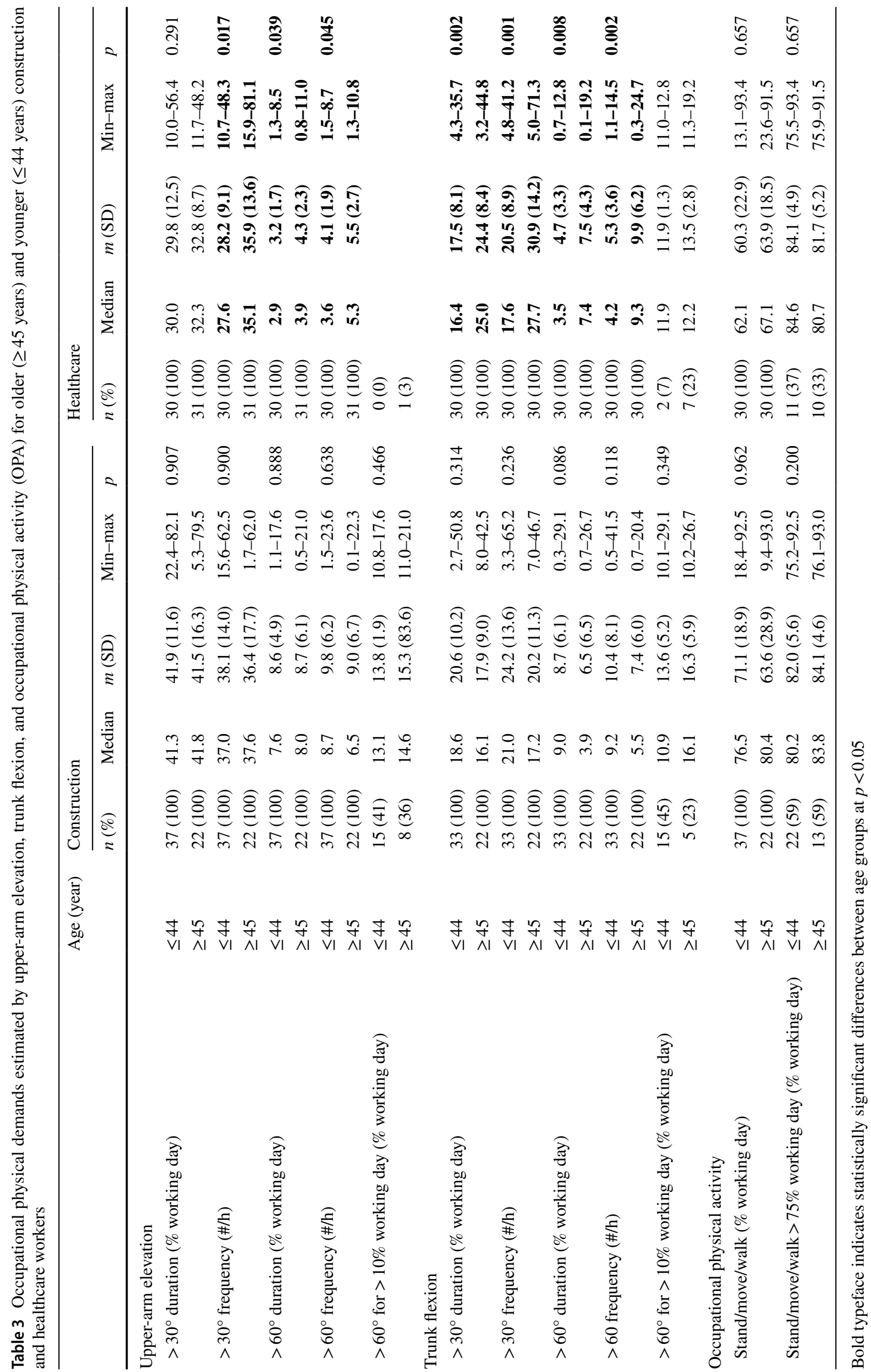




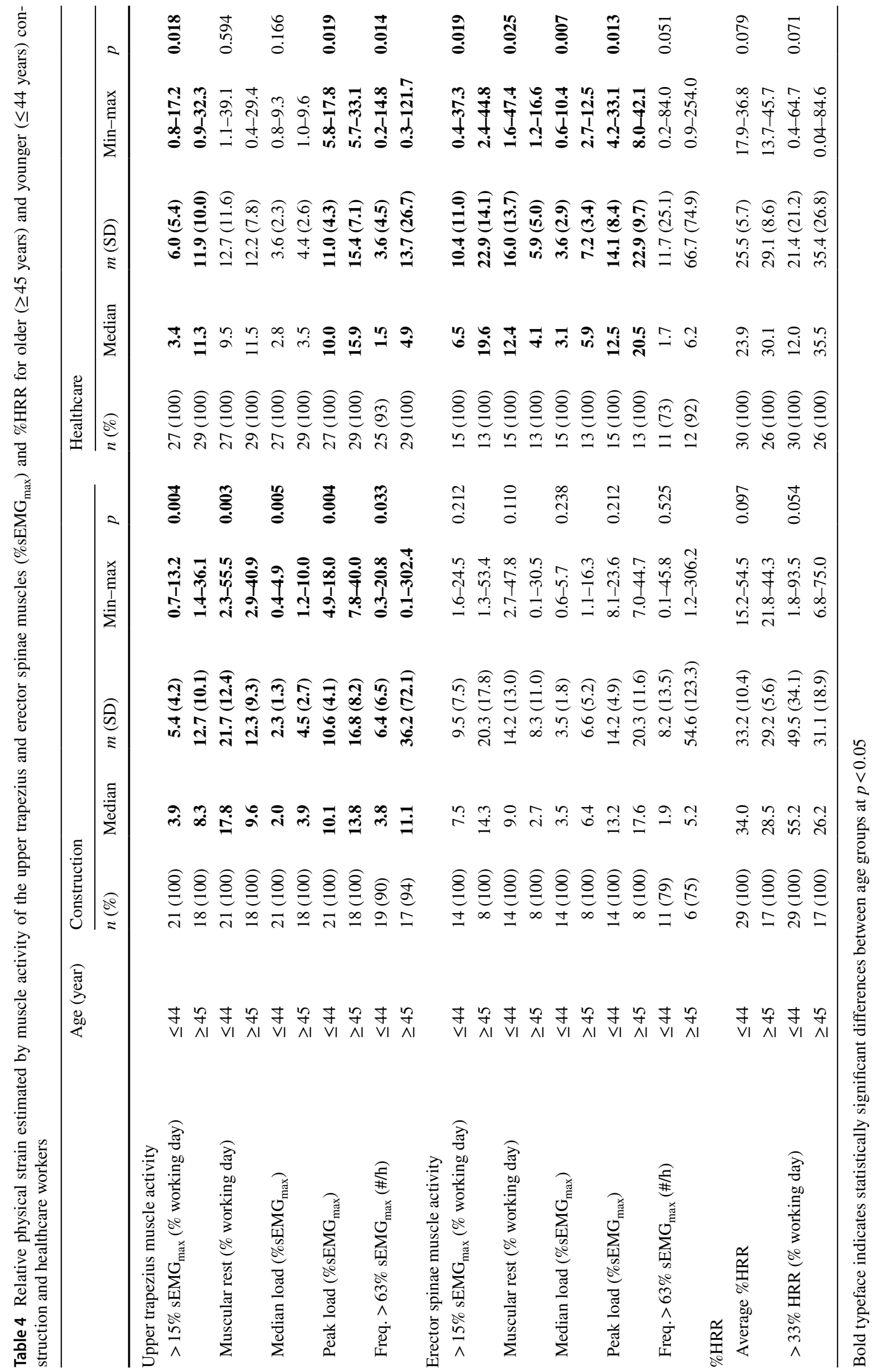


Table 5 Age modification of the associations between arm elevation $>60^{\circ}$ and shoulder strength, between trunk flexion $>60^{\circ}$ and back strength, and between occupational physical activity and $\mathrm{VO}_{2 \max }$, respectively

\begin{tabular}{|c|c|c|c|}
\hline \multirow[t]{3}{*}{ Construction $(n=62)$} & $\begin{array}{l}\text { Arm elevation }>60^{\circ}(\% \text { working } \\
\text { day })^{\mathrm{a}}\end{array}$ & Trunk flexion $>60^{\circ}(\% \text { working day })^{\mathrm{b}}$ & 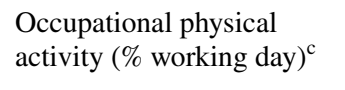 \\
\hline & $\operatorname{Exp}(b)(95 \% \mathrm{CI})$ & $b(95 \% \mathrm{CI})$ & $\operatorname{Sqrt}(b)(95 \% \mathrm{CI})$ \\
\hline & $n=59$ & $n=53$ & $n=55$ \\
\hline \multicolumn{4}{|l|}{ Crude model } \\
\hline Capacity indicator & $1.00(0.96-1.04)$ & $0.04(-0.10$ to 0.18$)$ & $-3.66(-9.19$ to 7.60$)$ \\
\hline \multicolumn{4}{|l|}{ Age } \\
\hline$\leq 44$ years & 1.00 & 0.00 & 0.00 \\
\hline$\geq 45$ years & $0.14(0.03-0.68)$ & $-6.72(-17.60$ to 4.15$)$ & $-89.59(-124.96$ to 20.95$)$ \\
\hline Capacity indicator $\times$ age & $1.07(1.01-1.12)$ & $0.11(-1.00$ to 0.33$)$ & $15.21(3.38-21.24)$ \\
\hline \multicolumn{4}{|l|}{ Adjusted model } \\
\hline Capacity indicator & $1.01(0.98-1.04)$ & & $5.83(-1.97$ to 8.47$)$ \\
\hline \multicolumn{4}{|l|}{ Age } \\
\hline$\leq 44$ years & 1.00 & & 0.00 \\
\hline$\geq 45$ years & $0.45(0.13-1.60)$ & & $-14.54(-66.13$ to 62.85$)$ \\
\hline Capacity indicator $\times$ age & $1.03(0.98-1.07)$ & & $4.52(-9.97$ to 11.84$)$ \\
\hline \multicolumn{4}{|l|}{ Level of work demands } \\
\hline Sedentary/light work & 1.00 & & 0.00 \\
\hline Heavy work & $3.43(2.30-5.11)$ & & $69.69(63.48-75.39)$ \\
\hline \multirow[t]{3}{*}{ Healthcare $(n=64)$} & $\begin{array}{l}\text { Arm elevation }>60^{\circ}(\% \text { working } \\
\text { day })^{\mathrm{a}}\end{array}$ & Trunk flexion $>60^{\circ}(\% \text { working day })^{b}$ & $\begin{array}{l}\text { Occupational physical } \\
\text { activity }(\% \text { working day })^{\mathrm{c}}\end{array}$ \\
\hline & $\operatorname{Exp}(b)(95 \% \mathrm{CI})$ & $b(95 \% \mathrm{CI})$ & $\operatorname{Sqrt}(b)(95 \% \mathrm{CI})$ \\
\hline & $n=61$ & $n=59$ & $n=56$ \\
\hline \multicolumn{4}{|l|}{ Crude model } \\
\hline Capacity indicator & $0.99(0.96-1.03)$ & $-0.02(-0.11$ to 0.07$)$ & $-5.92(-11.23$ to 7.48$)$ \\
\hline \multicolumn{4}{|l|}{ Age } \\
\hline$\leq 44$ years & 1.00 & 0.00 & 0.00 \\
\hline$\geq 45$ years & $0.81(0.34-1.92)$ & $3.54(-1.52$ to 8.59$)$ & $40.34(-68.06$ to 88.81$)$ \\
\hline Capacity indicator $\times$ age & $1.03(0.98-1.08)$ & $-0.03(-0.18$ to 0.12$)$ & $-6.38(-15.54$ to 12.65$)$ \\
\hline
\end{tabular}

Bold typeface indicates statistically significant differences between age groups at $\mathrm{p}<0.05$

${ }^{\mathrm{a}}$ Capacity indicator for arm elevation $>60^{\circ}$ is shoulder strength $(\mathrm{kg})$

${ }^{\mathrm{b}}$ Capacity indicator for trunk flexion $>60^{\circ}$ is back strength $(\mathrm{kg})$

${ }^{\mathrm{c}}$ Capacity indicator for occupational physical activity is aerobic capacity $\left(V_{2 \max }\right)$

Age-related differences in relative physical strain depended on the sector. Among healthcare workers, older employees worked at higher levels of relative strain; this is in line with their higher self-reported exertion levels. Among construction workers, older employees, specifically those with heavier work demands, worked at a lower percentage of their heart rate reserve yet with an unfavourable muscle activity pattern. This paradox may suggest that older construction workers had strategies to reduce some, but not all heavier physical demands. Such strategies may include delegating heavier tasks to younger workers, as suggested by the tendency for older workers to have less trunk flexion and the unequal treatment reported by younger construction workers. Nevertheless, the unfavourable muscle activity pattern seen among older employees in both sectors, suggests that their tasks were more strenuous for them than the tasks of the younger workers. Maintaining muscular strength with age may not be sufficient to protect against a potential detrimental muscle activity pattern; therefore, physical demands may need to be reduced for older employees to reduce the risk for musculoskeletal disorders.

At a group level, our findings suggest that the older workers in our study may have had a poorer balance between capacity and demands than the younger workers, e.g., older workers had lower aerobic capacity yet similar occupational physical activity compared to their younger colleagues. This is in line with previous hypotheses and expectations (de Zwart et al. 1995; Holtermann et al. 2018). However, at an 

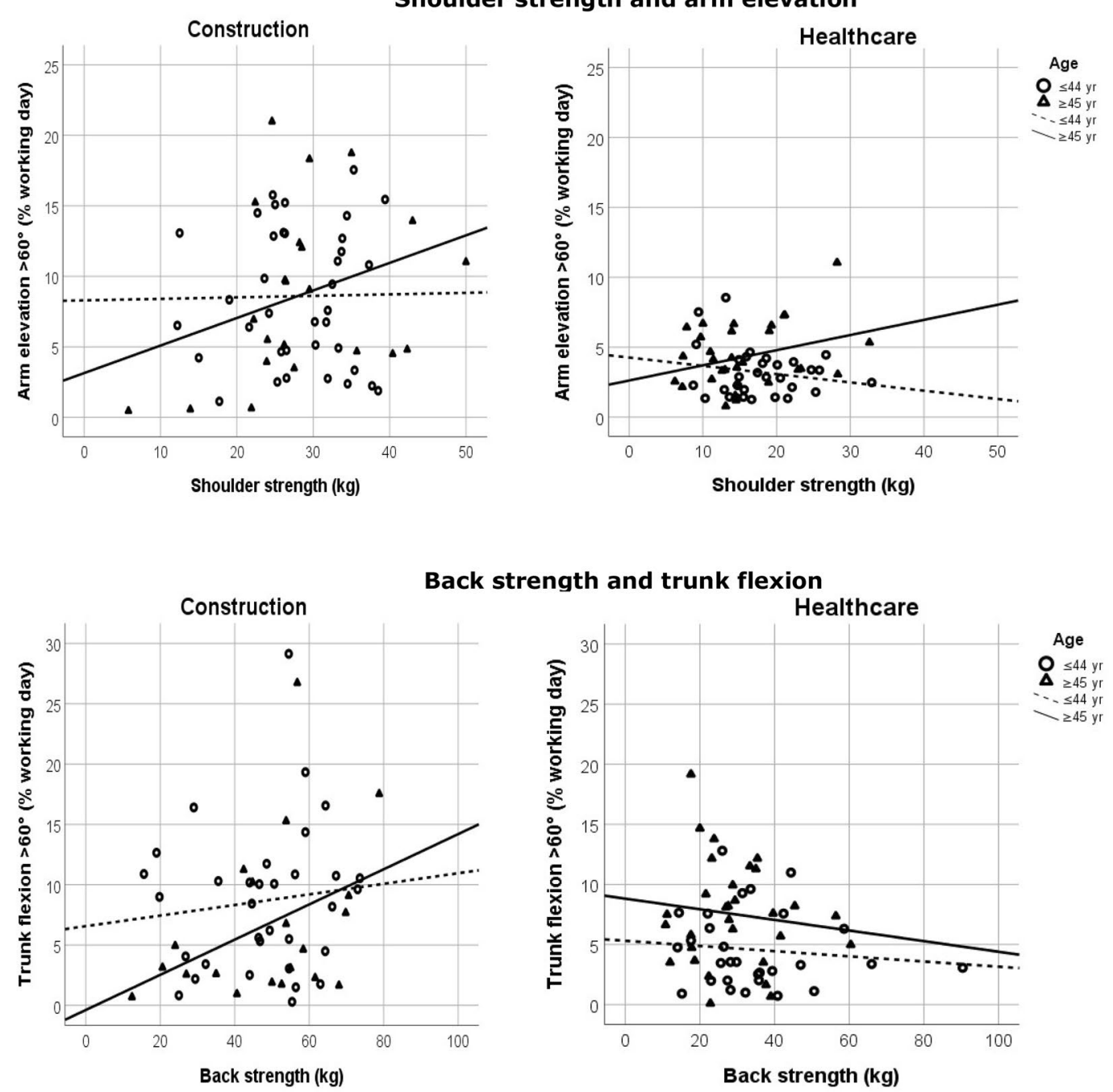

Aerobic capacity and occupational physical activity (OPA)
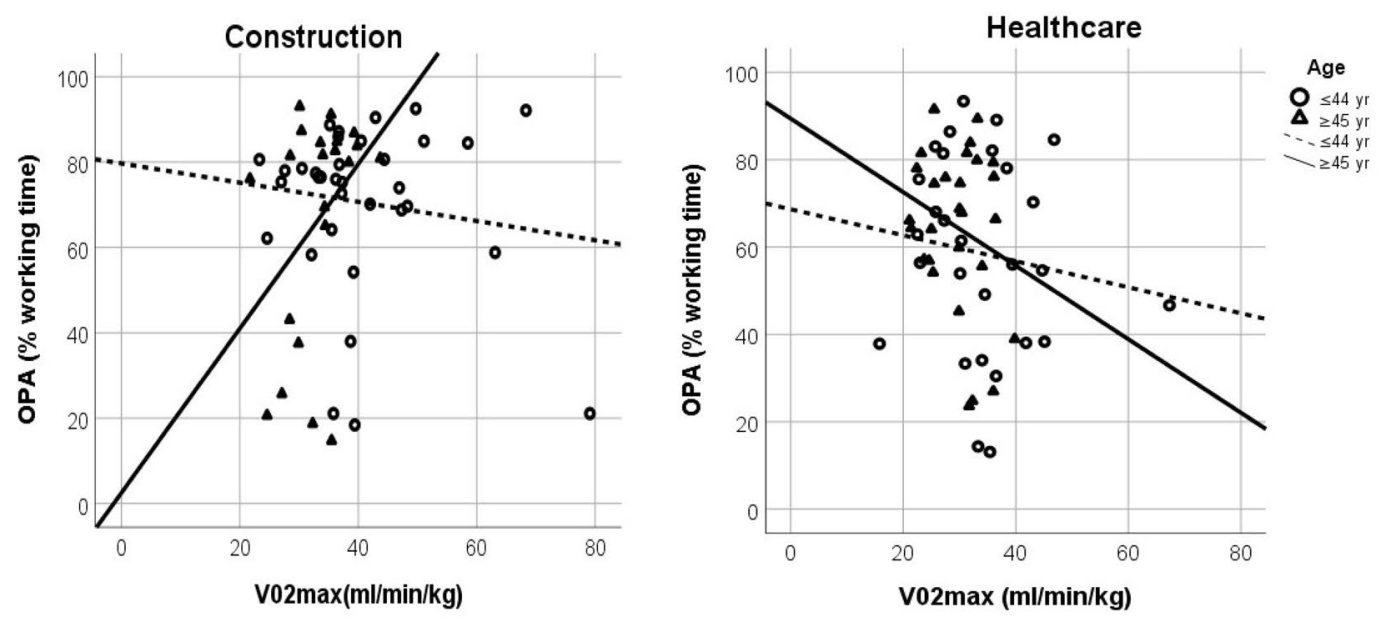

Fig. 1 The age-dependent associations between shoulder strength and arm elevation $>60^{\circ}$, between back strength and trunk flexion $>$ $60^{\circ}$, and between occupational physical activity and aerobic capacity

$\left(V \mathrm{O}_{2 \max }\right)$ among construction workers (left column) and healthcare workers (right column) 
individual level, our findings show a different association. At an individual level, the balance was likely better for older than younger construction workers, while it is similar for older and younger healthcare workers. Among the construction employees, this indicates that older workers with lower capacity had lower demands, while those with higher capacity had higher demands. In comparison, younger construction workers seemed to have similar demands, irrespective of capacity (Fig. 1). Older and younger healthcare workers too, seemed to have similar demands irrespective of capacity (Fig. 1).

The 'better' balance for older construction workers and 'similar' balance for older healthcare workers in our sample may be interpreted in a number of ways. First, the fact that the level of physical work demands (DOT) explained the better balance for construction workers, may suggest that the overrepresentation of older construction workers in our sample with sedentary/light work (who also had lower capacity) is due to a possibility to move into sedentary/light work (e.g., supervisors or project leaders) with age. Moving into lighter work may be a way to meet previously reported needs to reduce physical demands to remain at work (Jebens et al. 2014). The similar balance for older and younger healthcare workers suggests that they may not have such opportunities. Second, a better and similar balance-rather than a poorer balance-may have been observed owing to a healthy worker survival effect. Potentially, the older employees were a selfselected sample who remained at work, because they had maintained their muscle strength and work ability. Further, the better or similar balance of older employees is compared to the balance of younger employees whose balance may not have been optimal: in both sectors younger workers seemed to have similar demands irrespective of capacity. Therefore, the comparison with younger workers did not provide insight into whether a 'better' or 'similar' balance is a 'good' balance or sufficient to maintain health and sustain work. All three interpretations argue for reducing occupational physical demands with age as a means to promote sustainable work participation.

\section{Strengths and limitations}

A major strength of this study is the use of objective measurements that eliminated recall and self-estimation bias. In addition, the simultaneous assessments of several indicators of occupational physical demands and relative physical strain provided a comprehensive description of the effects of physically demanding occupations on aging employees. A limitation of this study was its cross-sectional design that did not follow the development of age-related changes over time. This may have given rise to a healthy worker survival effect among older employees that may have led to an overestimation of physical capacity and an underestimation of relative physical strain. However, this only supports the need to address the level of occupational physical demands to avoid premature dropout from the labour market. Furthermore, the sample size was relatively small and not randomly selected; therefore, the findings need to be interpreted with caution. However, a small sample size enabled several simultaneous objective measurements of occupational exposures and a close follow-up of each participant throughout their working day. Another limitation is the loss of data, specifically for the lower back sEMG assessments owing to electrodes falling off during a warm, sweaty summer. This likely led to power problems in the statistical analysis among construction workers; however, the substantial differences between the age groups may still be related to an increased risk for fatigue and musculoskeletal disorders.

\section{Future research}

The high level of occupational physical demands and relative physical strain among older employees in our sample urges future research to identify ways in which work can be organised that reduces physical demands and relative strain among older employees. In doing so, it should be recognised that similar exposure limits may have stronger effects on health among older than younger employees (Burr et al. 2017); therefore, dose-response relationships in relation to age should be identified. When assessing capacity, future studies may consider using dynamic capacity test, such as used in functional capacity assessments, in addition to the isometric capacity tests used in the present study. Where isometric tests determine the individual's general physical capacity, dynamic functional capacity tests reflect specific task demands more precisely. Moreover, future studies are suggested to assess physical capacity, occupational physical demands, and relative physical strain repeatedly over time to gain insight into how these variables change in the same individuals over time.

\section{Concluding remarks}

At a group level, when compared to younger workers, older employees had similar physical strength, yet lower aerobic capacity; however, the occupational physical demands were similar or higher. The muscle activity pattern was unfavourable for older healthcare workers, and percentage heart rate reserve had a tendency to be higher. For older construction workers, the muscle activity pattern was unfavourable, while percentage heart rate reserve had a tendency to be lower. At an individual level, the better balance between capacity and demands for older construction workers suggests that they may have opportunities to cope with the heavy physical demands. The similar balance for older and younger 
healthcare workers suggests that older healthcare workers may not have such opportunities. Overall, the level of occupational demands and relative physical strain, and the balance between capacity and demands among older employees, argue for reducing occupational physical demands with age as an incentive to promote sustainable work participation.

Acknowledgements We would like to thank all participants of this project for their time and effort. We would further like to thank Rune Madsen at the National Institute of Occupational Health in Oslo for assisting with data preparation. We are also grateful for the input concerning project design offered by Mikael Forsman, Andreas Holtermann, and Svend Erik Mathiassen.

Author contributions LKL, MK, MW, SK, and BV designed the study. LKL, MK, and MW participated in data collection. SLM performed the data analysis and drafted the manuscript. All authors interpreted the results, critically revised the manuscript for intellectual content, and approved the final version.

\section{Compliance with ethical standards}

Conflict of interest This project was funded by The Research Council of Norway, project number 218358. The Research Council of Norway did not have any role in the study design, the collection, analysis and interpretation of the data, nor in the writing of the manuscript or the decision to submit this paper for publication. The authors declare that they have no conflict of interest.

Ethical approval The study was approved by The Regional Committee for Medical and Health Research Ethics in Norway (2014/138/REK south-east D). All procedures of the study were in accordance with the ethical standards of the national research committee and with the 1964 Helsinki declaration and its later amendments or comparable ethical standards. Informed consent was obtained from all individual participants included in the study.

Open Access This article is distributed under the terms of the Creative Commons Attribution 4.0 International License (http://creativeco mmons.org/licenses/by/4.0/), which permits unrestricted use, distribution, and reproduction in any medium, provided you give appropriate credit to the original author(s) and the source, provide a link to the Creative Commons license, and indicate if changes were made.

\section{References}

Ahlstrom L, Grimby-Ekman A, Hagberg M, Dellve L (2010) The work ability index and single-item question: associations with sick leave, symptoms, and health-a prospective study of women on long-term sick leave. Scand J Work Environ Health 36(5):404-412

Aittomaki A, Lahelma E, Roos E, Leino-Arjas P, Martikainen P (2005) Gender differences in the association of age with physical workload and functioning. Occup Environ Med 62(2):95-100. https:// doi.org/10.1136/oem.2004.014035

Anton D, Cook TM, Rosecrance JC, Merlino LA (2003) Method for quantitatively assessing physical risk factors during variable noncyclic work. Scand J Work Environ Health 29(5):354-362

Astrand PO (2003) Textbook of work physiology: physiological bases of exercise, 4 th edn. Human Kinetics, Champaign
Bieringsorensen F (1984) Physical measurements as risk indicators for low-back trouble over a one-year period. Spine 9(2):106-119. https://doi.org/10.1097/00007632-198403000-00002

Brighenti-Zogg S et al (2016) Physical workload and work capacity across occupational groups. PLoS One 11(5):e0154073. https:// doi.org/10.1371/journal.pone.0154073

Burr H, Pohrt A, Rugulies R, Holtermann A, Hasselhorn HM (2017) Does age modify the association between physical work demands and deterioration of self-rated general health? Scand J Work Env Hea 43(3):241-249. https://doi.org/10.5271/sjweh .3625

Coenen P, Douwes M, van den Heuvel S, Bosch T (2016) Towards exposure limits for working postures and musculoskeletal symptoms - a prospective cohort study. Ergonomics 59(9):1182-1192. https://doi.org/10.1080/00140139.2015.1130862

Dallner M et al (2000) Validation of the General Nordic Questionnaire (QPSNordic) for psychological and social factors at work. Nordic Council of Ministers, Nord

Davis KG, Kotowski SE (2015) Prevalence of musculoskeletal disorders for nurses in hospitals, long-term care facilities, and home health care: a comprehensive review. Hum Fact 57(5):754-792. https://doi.org/10.1177/0018720815581933

de Zwart BC, Frings-Dresen MH, van Dijk FJ (1995) Physical workload and the aging worker: a review of the literature. Int Arch Occup Environ Health 68(1):1-12

Gall B, Parkhouse W (2004) Changes in physical capacity as a function of age in heavy manual work. Ergonomics 47(6):671-687. https ://doi.org/10.1080/00140130410001658691

Gupta N et al (2014) Face validity of the single work ability item: comparison with objectively measured heart rate reserve over several days. Int J Environ Res Public Health 11(5):5333-5348. https:// doi.org/10.3390/ijerph110505333

Hamberg-van Reenen HH, van der Beek AJ, Blatter BM, van Mechelen W, Bongers PM (2009) Age-related differences in muscular capacity among workers. Int Arch Occup Environ Health 82(9):11151121. https://doi.org/10.1007/s00420-009-0407-8

Holtermann A, Krause N, van der Beek AJ, Straker L (2018) The physical activity paradox: six reasons why occupational physical activity (OPA) does not confer the cardiovascular health benefits that leisure time physical activity does. Br J Sports Med 52(3):149_ 150. https://doi.org/10.1136/bjsports-2017-097965

Jarvholm B, Stattin M, Robroek SJ, Janlert U, Karlsson B, Burdorf A (2014) Heavy work and disability pension-a long term follow-up of Swedish construction workers. Scand J Work Environ Health 40(4):335-342. https://doi.org/10.5271/sjweh.3413

Jebens E, Medbo JI, Knutsen O, Mamen A, Veiersted KB (2014) Association between perceived present working conditions and demands versus attitude to early retirement among construction workers. Work 48(2):217-228. https://doi.org/10.3233/WOR141863

Jebens E, Mamen A, Medbo JI, Knudsen O, Veiersted KB (2015) Are elderly construction workers sufficiently fit for heavy manual labour? Ergonomics 58(3):450-462. https://doi. org/10.1080/00140139.2014.977828

Jensen LD, Ryom PK, Christensen MV, Andersen JH (2012) Differences in risk factors for voluntary early retirement and disability pension: a 15-year follow-up in a cohort of nurses' aides. BMJ Open. https://doi.org/10.1136/bmjopen-2012-000991

Koch M, Lunde LK, Gjulem T, Knardahl S, Veiersted KB (2016) Validity of questionnaire and representativeness of objective methods for measurements of mechanical exposures in construction and health care work. PLoS One 11(9):e0162881. https://doi. org/10.1371/journal.pone.0162881

Kuorinka I et al (1987) Standardised Nordic questionnaires for the analysis of musculoskeletal symptoms. Appl Ergon 18(3):233-237 
Labriola M, Feveile H, Christensen KB, Stroyer J, Lund T (2009) The impact of ergonomic work environment exposures on the risk of disability pension: prospective results from DWECS/DREAM. Ergonomics 52(11):1419-1422. https://doi.org/10.1080/00140 130903067771

Lunde LK et al (2014) Musculoskeletal health and work ability in physically demanding occupations: study protocol for a prospective field study on construction and health care workers. BMC Public Health 14:1075. https://doi.org/10.1186/1471-2458-14-1075

Mathiassen SE, Winkel J (1991) Quantifying variation in physical load using exposure-vs-time data. Ergonomics 34(12):1455-1468. https://doi.org/10.1080/00140139108964889

Mathiassen SE, Winkel J, Hagg GM (1995) Normalization of surface EMG amplitude from the upper trapezius muscle in ergonomic studies-a review. J Electromyogr Kinesiol 5(4):197-226

Nygard CH, Luopajarvi T, Ilmarinen J (1991) Musculoskeletal capacity and its changes among aging municipal employees in different work categories. Scand J Work Environ Health 17(Suppl 1):110-117

Oude Hengel KM, Blatter BM, Geuskens GA, Koppes LL, Bongers PM (2012) Factors associated with the ability and willingness to continue working until the age of 65 in construction workers. Int Arch Occup Environ Health 85(7):783-790. https://doi. org/10.1007/s00420-011-0719-3

Rasmussen CD, Andersen LL, Clausen T, Stroyer J, Jorgensen MB, Holtermann A (2015) Physical capacity and risk for long-term sickness absence: a prospective cohort study among 8664 female health care workers. J Occup Environ Med 57(5):526-530. https ://doi.org/10.1097/JOM.0000000000000395

Schibye B, Hansen AF, Sogaard K, Christensen H (2001) Aerobic power and muscle strength among young and elderly workers with and without physically demanding work tasks. Appl Ergon 32(5):425-431

Skotte J, Korshoj M, Kristiansen J, Hanisch C, Holtermann A (2014) Detection of physical activity types using triaxial accelerometers. J Phys Act Health 11(1):76-84. https:// doi.org/10.1123/jpah.2011-0347

Soer R, Brouwer S, Geertzen JH, van der Schans CP, Groothoff JW, Reneman MF (2012) Decline of functional capacity in healthy aging workers. Arch Phys Med Rehabil 93(12):2326-2332. https ://doi.org/10.1016/j.apmr.2012.07.009

Sundstrup E et al (2018) Retrospectively assessed physical work environment during working life and risk of sickness absence and labour market exit among older workers. Occup Environ Med 75(2):114-123. https://doi.org/10.1136/oemed-2016-104279

Tanaka H, Monahan KD, Seals DR (2001) Age-predicted maximal heart rate revisited. J Am Coll Cardiol 37(1):153-156

Tonnon SC et al (2017) The employer perspective on sustainable employability in the construction industry. J Occup Environ Med 59(1):85-91. https://doi.org/10.1097/JOM.0000000000000913

Torgen M, Punnett L, Alfredsson L, Kilbom A (1999) Physical capacity in relation to present and past physical load at work: a study of 484 men and women aged 41 to 58 years. Am J Ind Med 36(3):388-400

U.S. Department of Labor EaTA (1991) Dictionary of Occupational Titles. In: U.S. Government Printing Office. https://www.oalj.dol. gov/LIBDOT.HTM. Accessed 15 Nov 2017

Umer W, Antwi-Afari MF, Li H, Szeto GPY, Wong AYL (2017) The prevalence of musculoskeletal symptoms in the construction industry: a systematic review and meta-analysis. Int Arch Occup Environ Health 91(2):125-144. https://doi.org/10.1007/s0042 0-017-1273-4

Veiersted KB, Westgaard RH, Andersen P (1993) Electromyographic evaluation of muscular work pattern as a predictor of trapezius myalgia. Scand J Work Environ Health 19(4):284-290

Ware JE Jr (2000) SF-36 health survey update. Spine (Phila Pa 1976) 25(24):3130-3139 